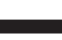

\title{
Permanently Hypoxic Cell Culture Yields Rat Bone Marrow Mesenchymal Cells with Higher Therapeutic Potential in the Treatment of Chronic Myocardial Infarction
}

\author{
Yihua Liu Lib,c $^{a}$ Xiaoxi Yang ${ }^{f}$ Pablo Maureira ${ }^{a, c} \quad$ Aude Falanga ${ }^{a}$ Vanessa Marie ${ }^{a}$ \\ Guillaume Gauchotte $^{\mathrm{d}}$ Sylvain Poussier ${ }^{\mathrm{e}}$ Frederique Groubatch ${ }^{\mathrm{a}}$ \\ Pierre-Yves Marie ${ }^{a, e} \quad$ Nguyen Tran ${ }^{a}$
}

aSchool of Surgery, INSERM U1116, Faculty of Medicine, University of Lorraine, France, Nancy, France; ${ }^{b}$ Department of cardiovascular surgery, Union Hospital, Tongji Medical College, Huazhong University of science and technology, Wuhan, China; 'Department of Cardiovascular Surgery, Nancy University Hospital, Nancy France; d Department of Pathology, Nancy University Hospital, France; ${ }^{e}$ Department of Nuclear Medicine, Nancy University Hospital, Nancy, France; 'Department of Radiation and Medical Oncology, Zhongnan Hospital, Wuhan University, Wuhan, China

\section{Key Words}

Hypoxic preconditioning $\bullet$ Mesenchymal stem cells $\bullet$ Chronic myocardial infarction $\bullet$ Myocardial viability • Angiogenesis

\begin{abstract}
Background: The mismatch between traditional in vitro cell culture conditions and targeted chronic hypoxic myocardial tissue could potentially hamper the therapeutic effects of implanted bone marrow mesenchymal stem cells (BMSCs). This study sought to address (i) the extent of change to BMSC biological characteristics in different in vitro culture conditions and (ii) the effectiveness of permanent hypoxic culture for cell therapy in treating chronic myocardial infarction (MI) in rats. Methods: rat BMSCs were harvested and cultured in normoxic (21\% $\left.\mathrm{O}_{2^{\prime}} \mathrm{n}=27\right)$ or hypoxic conditions $\left(5 \% \mathrm{O}_{2^{\prime}} \mathrm{n}=27\right)$ until Passage 4 (P4). Cell growth tests, flow cytometry, and Bio-Plex assays were conducted to explore variations in the cell proliferation, phenotype, and cytokine expression, respectively. In the in vivo set-up, P3-BMSCs cultured in normoxia $(n=6)$ or hypoxia $(n=6)$ were intramyocardially injected into rat hearts that had previously experienced 1-month-old MI. The impact of cell therapy on cardiac segmental viability and hemodynamic performance was assessed 1 month later by 2-Deoxy-2[ $\left.{ }^{18} \mathrm{~F}\right]$ fluoroD-glucose $\left({ }^{18} \mathrm{~F}-\mathrm{FDG}\right)$ positron emission tomography (PET) imaging and pressure-volume catheter, respectively. Additional histomorphological examinations were conducted to evaluate inflammation, fibrosis, and neovascularization. Results: Hypoxic preconditioning significantly enhanced rat BMSC clonogenic potential and proliferation without altering the multipotency.

Y. Liu and X. Yang contributed equally to this work.


Different profiles of inflammatory, fibrotic, and angiogenic cytokine secretion were also documented, with a marked correlation observed between in vitro and in vivo proangiogenic cytokine expression and tissue neovessels. Hypoxic-preconditioned cells presented a beneficial effect on the myocardial viability of infarct segments and intrinsic contractility. Conclusion: Hypoxic-preconditioned BMSCs were able to benefit myocardial perfusion and contractility, probably by modulating the inflammation and promoting angiogenesis.

\section{Introduction}

(C) 2017 The Author(s)

Published by S. Karger AG, Basel

Chronic ischemic heart failure continues to pose an unequivocal challenge to the health care systems around the world owing to the structural complexity of cardiac tissue, as well as the polymorphism of tissue degradation inherent to the chronic phase of myocardial infarction (MI) that is still refractory to conventional treatments. In recent decades, cell-based therapies including administering autologous or allogenous bone marrow mesenchymal stem cells (BMSCs) have rapidly emerged as a promising approach to promote cardiac repair and regeneration following ischemic insults $[1,2]$. While early experimental studies and clinical trials have shown encouraging results, it cannot be denied that the reported therapeutic effects of BMSCs are largely suboptimal [3, 4].

The main cause for concern is the low engraftment and survival rates, since the literature reports that merely less than $1 \%$ of BMSCs typically survive for 4 days following transplantation [5]. One of the possible explanations for cell engraftment failure is related to the mismatch between conventional in vitro normoxic culture for cell expansion and the severely hypoxic infarcted area that is targeted [6, 7]. Furthermore, the BMSCs niche is found to be "physiologically" hypoxic [8]. This condition is recognized to be crucial for the maintenance of self-renewal capacity, undifferentiated state, and genetic stability of the BMSCs [6, 9-12]. The pleiotropic effects of hypoxic conditions on BMSC behaviors have been intensively investigated in vitro in the last 20 years. However, there has been a great heterogeneity among experimental protocols differing in duration of exposure $(0 \sim 72 \mathrm{~h}$ versus permanent) and severity of hypoxia (oxygen concentration varying from 0 to $5 \%$ ) [7, $10,13-18]$. It's even speculated that the hypoxic effects on BMSC behaviors are biphasic with acute short-term oxygen depletion provoking apoptosis while long-term hypoxic exposure being helpful for the maintenance of an undifferentiated state [19].

Despite intensive investigations, the mechanism of action of BMSCs has not yet been deciphered. The paracrine effects including immuno-modulation, secretion of growth factors, cytokines, anti-fibrotic, anti-apoptotic and angiogenic factors, instead of transdifferentiation into cardiomyocytes, are recognized as the main mechanism underlying cardiac repair with cell therapy [20-25]. Besides the well-documented beneficial effects of several growth factors such as vascular epithelial growth factor (VEGF), several cytokines expressed by BMSCs have been supposed to mediate the immunomodulation effect in cell-based therapy, including IFN- $\gamma$, TNF- $\alpha$ and IL-6[21, 26]. It has been demonstrated that hypoxic culture enhances the secretion of paracrine factors and promotes therapeutic effects of BMSCs [19]. A microarray study revealed that hypoxic BMSCs increase the expression of several growth factors involved in cell proliferation, survival and angiogenesis, and the changes in cytokine expression profile are related to the improvement of therapeutic efficacy in the treatment of acute MI[14]. However, the studies are still scarce regarding impacts of long-term hypoxic preconditioning on BMSC therapeutic potential in the management of chronic MI.

We designed this study with the aim to address the two following issues: (i) the extent of changes to biological characteristics of BMSCs under long-term (1 month) normoxic $\left(\mathrm{O}_{2} 21 \%\right)$ or hypoxic $\left(\mathrm{O}_{2} 5 \%\right)$ culture and (ii) the impacts of such culture condition on the therapeutic potential in treating chronic MI. We believe that permanent ex vivo hypoxic culture is more relevant to the environment of BMSC niches in situ where the oxygen concentration is reported to be $2-9 \%[5,8]$. 


\section{Cellular Physiology Cell Physiol Biochem 2017;44:1064-1077 \begin{tabular}{l|l|l} 
and Biochemistry Published online: November 24, 2017 & $\begin{array}{l}\text { (c) } 2017 \text { The Author(s). Published by S. Karger AG, Basel } \\
\text { www.karger.com/cpb }\end{array}$ \\
\hline
\end{tabular}}

Liu et al.: Hypoxia and Cellular Therapy

\section{Materials and Methods}

\section{Animals}

This study was conducted using Wistar rats (4-6 months old, with an initial body-weight of 350g-450g). All experimental procedures were in accordance with our local ethics committee and with the regulations of the Animal Welfare Act of the National Institutes of Health (NIH) Guide for the Care and Use of Laboratory Animals (NIH Publication no. 85-23, Revised 1996).

\section{Anesthesia}

For invasive surgical procedures and examinations, animal anesthesia was achieved by inhalation of isoflurane (2-3 vol\%) and oxygen (2L/min) via tracheal intubation connected to mechanical ventilation (Minerve, Esternay, France).

\section{BMSCs harvest and culture}

As previously described $[17,27]$, the bone marrow was harvested $(n=27)$ through tibial puncture using a 28-gauge needle. The obtained bone marrow solution was centrifuged at $400 \mathrm{~g}$ for 10 minutes. Cell pellets were resuspended in Iscove's modified Dulbecco's medium, IMDM (Gibco, USA), supplemented with 10\% fetal bovine serum (Gibco, USA), 0.1 mmol. $\mathrm{L}^{-1} \beta$-mercaptoethanol (Sigma, USA) and 1\% antibiotics (penicillin and streptomycin) (Gibco, USA). They were then cultivated in $25 \mathrm{~cm}^{2}$ flasks at $37^{\circ} \mathrm{C}$ in a humidified atmosphere containing $5 \% \mathrm{CO}_{2}$. On Day 4, the non-adherent cells were washed out with phosphate buffered saline (PBS) and the adherent cells were further expanded until reaching 80\% confluence. They were then passaged using $0.25 \%$ trypsin and expanded in $75 \mathrm{~cm}^{2}$ flasks at a density of $6 \times 10^{3}$ cells per $\mathrm{cm}^{2}$. From Passage 1 (P1), the BMSCs derived from each rat were divided into two groups, one cultivated in normoxic conditions (21\% oxygen tension) and the other in hypoxic conditions (5\% oxygen tension), until P4. The hypoxic environment was achieved using a dedicated incubator $\mathrm{C}_{2}$ Control InVivo Glove Box, Coy Lab, Michigan, USA). The oxygen concentration in the hypoxic incubator was maintained at 5\% with a residual gas mixture composed of 5\% carbon dioxide and balanced nitrogen. For cell therapy, the BMSCs of both conditions were harvested at P3 and prepared for cell therapy.

\section{Establishment and evaluation of myocardial infarction}

At the same time as harvesting the bone marrow, myocardial infarction (MI) was induced in 27 animals by means of permanent coronary ligation, as previously described [27]. In brief, the hearts were accessed via left anterolateral thoracotomy through the fifth intercostal space. The left anterior descending artery was ligated by applying a 7/0 Prolene suture (Ethicon, Somerville, NJ). Analgesic agent, namely Lidocain (Panpharma, France), and antibiotics Cefamendole (Panpharma, France) were administered intramuscularly after incision closure. The surgical success rate was $67 \%(18 / 27)$.

One month following MI, an original ${ }^{18} \mathrm{~F}$-FDG-PET imaging technique was performed in the rats to assess the infarct areas and the baseline LV function, using a dedicated small-animal PET system (Inveon, Siemens, Knoxville, TN, USA). Quantitative analysis was performed using the 17-segment LV division method by means of QGS software. The segments with low mean myocardial FDG activity $(<70 \%$ of maximal voxel value) were defined as exhibiting infarct. The rats were equally divided into control group ( $\mathrm{n}=6)$, normoxic group ( $n=6)$ and hypoxic group $(n=6)$ according to their infarct area, each group receiving saline, BMSCs cultured in normoxia, or BMSCs cultured in hypoxia, respectively.

\section{Cell characterization}

The clonogenic potential of the BMSCs was assessed by counting the number of fibroblast colonyforming units (CFU-f) that has been stained with methylene blue and visualized by phase-contrast microscopy. The population-doubling time was calculated by taking a daily count of the cells in a Thoma counting chamber, and BMSC immunophenotype characterization was achieved by analyzing the surface markers of stem cells by means of flow cytometry. The BMSCs were characterized as CD44+, CD90+, CD34and CD45- as described previously $(28,29)$. Briefly, BMSCs $\left(1 \times 10^{5}\right.$ cells $\left./ 500 \mu \mathrm{L}\right)$ were incubated for 20 minutes with $10 \mu \mathrm{L}$ mononclonal antibodies against specific membrane markers (anti-CD44-FITC, clone 329, Antigenix America; anti-CD90-FITC, clone OX-7, Caltag; anti-CD34-FITC, clone ICO115, Santa Cruz; and anti-CD45-FITC, clone OX-1, Caltag) or isotypic IgG1 control (clone OX-1, Caltag Medsystems Ltd, UK), they 


\section{Cellular Physiology Cell Physiol Biochem 2017;44:1064-1077 \begin{tabular}{l|l|l} 
and Biochemistry $10.1159 / 000485406$ & $\begin{array}{l}\text { C) } 2017 \text { The Author(s). Published by S. Karger AG, Basel } \\
\text { www.karger.com/cpb }\end{array}$
\end{tabular}}

Liu et al.: Hypoxia and Cellular Therapy

were then processed through a FACS Calibur system (Becton Dickinson, San Jose, CA, USA) according to the manufacturer's instruction. The data were analyzed with Cellquest ${ }^{\mathrm{TM}}$ Pro Software (BD, San Jose, CA, USA).

\section{Differentiation assays}

The differentiation potential of BMSCs into adipocytes and osteocytes was evaluated as previously described(26). In brief, BMSCs were cultivated in either normoxia or hypoxia until P2 then exposed to osteogenic or adipogenic lineage-specific media, for 21 days for adipocyte differentiation and 28 days for osteocyte. The differentiated cells were characterized by means of histology staining using Oil Red 0 for adipocytes and Alizarine red for osteocytes.

The evaluation of differentiation into chondrocytes was performed after pellet culture. The P2BMSCs preconditioned with either normoxia or hypoxia were cultured in a $15 \mathrm{~mL}$ polypropylene tube in chondrogenic lineage-specific media for 28 days. The pellets were fixated with incubation for $24 \mathrm{~h}$ in Picric acid-Formalin solution. The differentiated cells were characterized by means of histology staining of the pellet slices, using Alcian blue and Kernechtrot dyes.

\section{Cell therapy}

One month following MI induction, the rats received an intra-myocardial injection of BMSCs cultivated in either normoxia or hypoxia, or physiological saline solution. The cells were conditioned in an insulin syringe $\left(2 \times 10^{6}\right.$ cells in $\left.50 \mu \mathrm{L}\right)$, and a single injection was administered via redo thoracotomy into the visually delineated fibrotic area of MI. One month after the BMSC implantation, another ${ }^{18} \mathrm{~F}$-FDG PET scan was performed to evaluate the impact of cell therapy on myocardial metabolism and function.

\section{Left ventricle pressure-volume (P-V) conductance catheter}

Four weeks following BMSC injection, the hemodynamic performance of all three groups' hearts was evaluated by means of P-V catheter (SPR-838, 2F, Millar Instruments Inc.). The hearts were accessed via the right carotid artery. We categorized our finding as "steady state", "hypertonic saline infusion", and "inferior vena cava occlusion" conditions, recorded and analyzed using the MPVS Ultra and Emka Technologies' Iox2 acquisition software (Millar Instruments Inc.).

\section{Bio-Plex analysis}

BMSC cytokine production and the cytokine levels in the infarct heart tissue were assessed by means of Bio-Plex analysis. Lysates taken from cultivated BMSCs and homogenates of heart tissue were prepared using a Bio-Plex cell lysis kit (Bio-Rad, France), and the extracted samples were analyzed with a multiplex rat cytokine assay (Bio-Plex Pro assays, Bio-Rad, France) in accordance with the manufacturer's instructions. The assessed parameters included tumor necrosis factor alpha (TNF- $\alpha$ ), granulocyte macrophage colonystimulating factor (GM-CSF), Interleukin (IL)-6, IL-10, vascular endothelial growth factor (VEGF) and transforming growth factor beta (TGF- $\beta 1$ ).

\section{Histo-morphological analysis}

Following formalin fixation and paraffin-embedding, $5 \mu \mathrm{m}$ sections of the heart tissues were stained with hematoxylin, eosin and saffron (HES) to evaluate the myocardial inflammatory infiltration and fibrosis. Myocardial inflammation was assessed by two pathologists blinded to this study, using the following semiquantitative score according to the intensity of inflammatory cells infiltration: 0 for absence of inflammation; 1 for mild inflammation; 2 for moderate inflammation; 3 for severe inflammation. Picrosirius red staining was performed to assess the total collagen content by measuring red stained areas over the field area by means of Image J software (http://imagej.nih.gov/), as previously reported(30). Immunohistochemical staining with anti-platelet endothelial cell adhesion molecule (PECAM-1, or CD31) antibody was also performed to evaluate neovessel formation. Vascular density was evaluated by counting the number of PECAM-1-positive micro-vessels in the infarct area, applying consecutive high-power fields $\left(0.237 \mathrm{~mm}^{2}\right)$.

\section{Statistics}

Data are presented as mean \pm standard deviation (SD). Comparisons between groups were achieved by means of unpaired Student's $t$-test, where appropriate. For ordinal data, such as the intensities of inflammation and infarcted segments, non-parametric Mann-Whitney U-test was used. A $p<0.05$ was 
Fig. 1. Cell proliferation and surface makers of BMSCs cultured in normoxic and hypoxic conditions. (A) CFU-f assay showed that the colony forming ability of BMSCs was enhanced in hypoxia. (B) The cell-population doubling time of BMSCs was significantly shorter when cultured in hypoxic condition. (C) Representative flow cytometry images of BMSCs cultured in hypoxia indicated CD90 to be a predominant surface marker (>95\%). (D) Flow cytometry revealed that rat BMSCs were characterized by $\mathrm{CD} 34(-)$, CD45(-), CD44(+), and CD90(+), the immunophynotype remained stable during the first 4 passages and comparable in both culture conditions. ${ }^{*} \mathrm{p}<0.05$

considered to denote statistical significance. Statistical analysis was performed using the SPSS software package V.24.0 (SPSS Inc., Chicago, USA).

\section{Results}

Hypoxia promoted BMSC clonogenic potential and cell proliferation

As shown in Fig. 1A, the clonogenic potential of BMSCs, evidenced by CFU-f assay in every cell passage, was strongly enhanced when cultured in hypoxia, compared with normoxia conditions (all $p<0.05$ ), while maximum clonogenicity was observed in P2 with both culture conditions. The BMSC proliferation (Fig. 1B) rates were also benefited by the hypoxic conditions, with a cell doubling time of $2.11 \pm 0.15$ days at $\mathrm{P} 1$ and $2.41 \pm 0.18$ days at $\mathrm{P} 4$, whereas the cell doubling time in normoxic condition was $3.17 \pm 0.23$ days at P1 and $3.64 \pm 0.28$ days at $\mathrm{P} 4$, both $p$ $<0.01$.

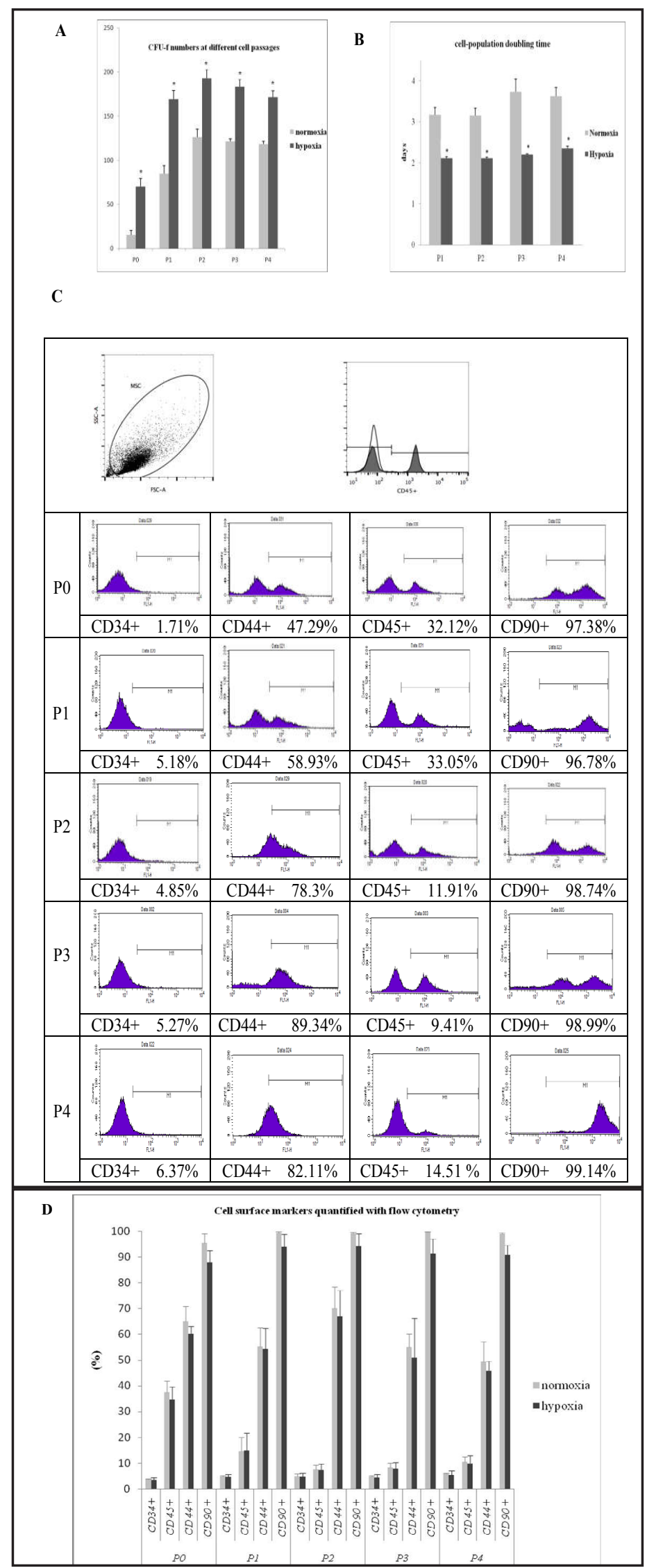


BMSC phenotype was assessed for each subculturing passage by analyzing the surface markers via flow cytometry (Fig. 1C, 1D). Similar profiles of BMSC phenotype in each passage were documented in both culture conditions, with BMSCs being positive for CD90 (>95\%) and for CD44 (>70\%), negative for CD34 (<5\%) and for CD45 (<7\%) since P2.

\section{Hypoxia maintained an undifferentiated state of BMSCs}

We performed different staining techniques (Oil Red 0, Alizarine red, and Alcian blue, Fig. 2), which revealed that the differentiation rates of the BMSCs into adipocytes, osteocytes, and chondrocytes were $47.2 \pm 6.8 \%, 86.3 \pm 8.2 \%$, and $17.0 \pm 3.7 \%$, respectively, in normoxic condition. This differed from those in hypoxic condition: $26.2 \pm 4.8 \%, 31.3 \pm 4.1 \%$, and $43.0 \pm 3.0 \%$, respectively (all $p<0.05$ ), indicating that hypoxia might have a more favorable influence on BMSC differentiation into chondrocytes, whereas normoxia promoted BMSC differentiation into osteocytes and adipocytes.

\section{Hypoxia-pretreated BMSCs enhanced myocardial perfusion}

As shown in Fig. 3A, pre-therapeutic PET imaging revealed comparable cardiac functional impairment and infarct area in all three groups. Following BMSC injection, an improvement in FDG uptake (Fig. 3B) in the infarct zone was observed in both the normoxia $(+3 \pm 3.2 \%)$ and hypoxia groups $(+5 \pm 5.8 \%)$, while the control group presented no improvement $(-1 \pm 3.5 \%)$.

Fig. 2. Photos and image illustrated the varied differentiation potential of BMSCs into adipocytes, osteocytes and chondrocytes demonstrated by Oil Red staining, Alizarin Red staining and Alcian Blue staining, respectively. Magnification was $\times 100$. The differentiation into adipocytes and osteocytes was favored by normoxia, while the differentiation into chondrocytes was favored by hypoxia. *, p $<0.05$ compared to Normoxia group.

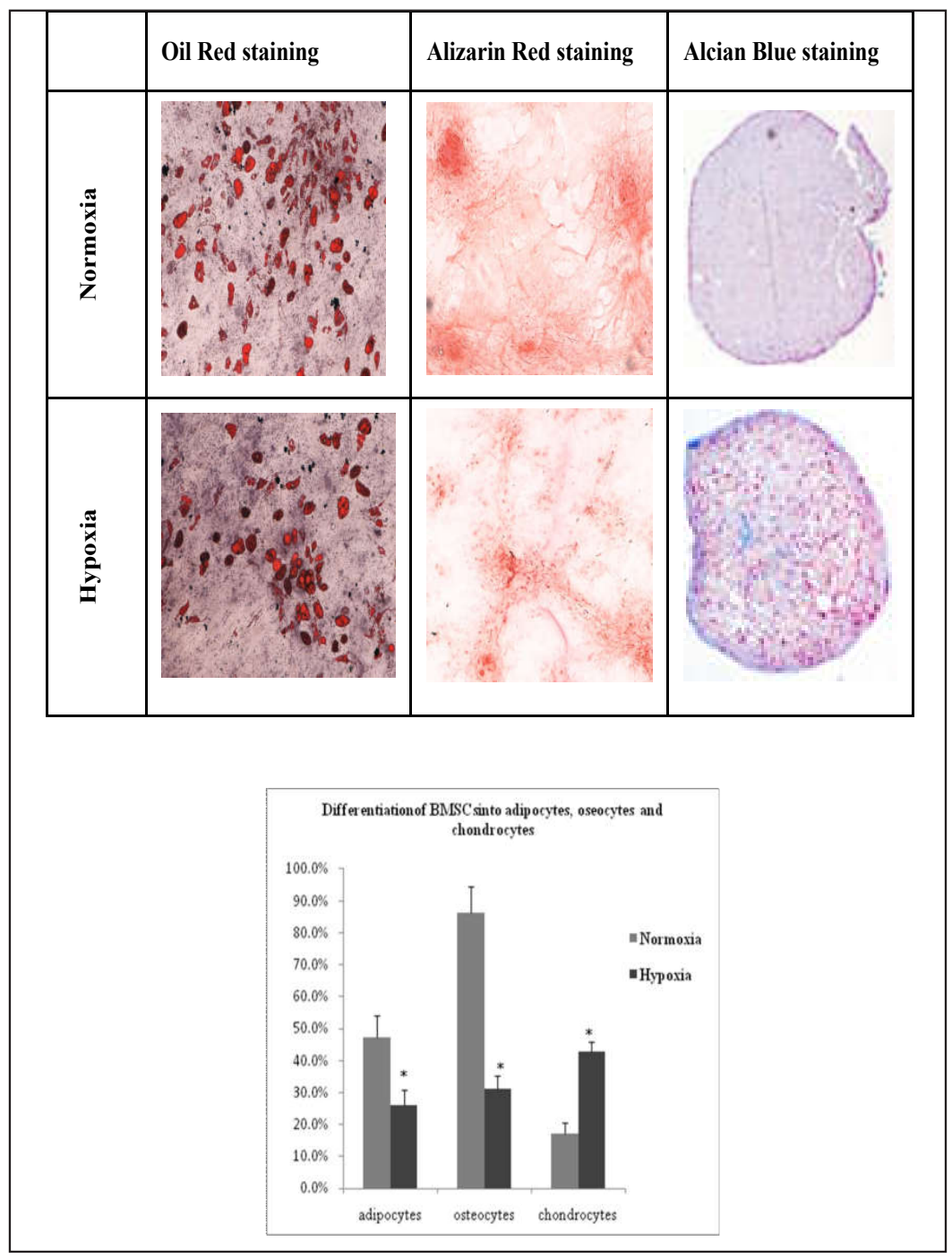


A similar change was observed in the reduction of infarct area (Fig. 3C) by $-0.2 \pm 1.2$, $0.6 \pm 1.0$ and $1.5 \pm 1.0$ segments in the control, normoxia, and hypoxia groups, respectively. However, these beneficial structural changes appeared to have no link to cardiac functional improvement, since the PET exams demonstrated no difference in left ventricular ejection fraction (Fig. 3D) either between groups, or between pre- and post-BMSC administration.

\section{Hypoxia-pretreated BMSCs improved myocardial intrinsic contractility}

Table 1 presents the hemodynamic parameters assessed by means of pressure-volume catheter in all three groups. In contrast to control group, the normoxia and hypoxia groups demonstrated a trend of improvement in several volume-dependent parameters, such as ejection fraction, cardiac output and stroke work $(p>0.05)$. However, the volume-independent parameters signifying the intrinsic contractility obtained with vena cave inferior occlusion $\left(\mathrm{dP} / \mathrm{dT}_{\text {max }} \mathrm{EDV}, \mathrm{PRSW}\right)$ were significantly best preserved in the hypoxia group out of the three.

\section{Hypoxia modified in vitro and in vivo cytokine expression spectrum}

Bio-Plex assay demonstrated oxygen tension in the culture medium to have a profound impact of on BMSC production of cytokines (Fig. 4). Compared to normoxic culture, hypoxic

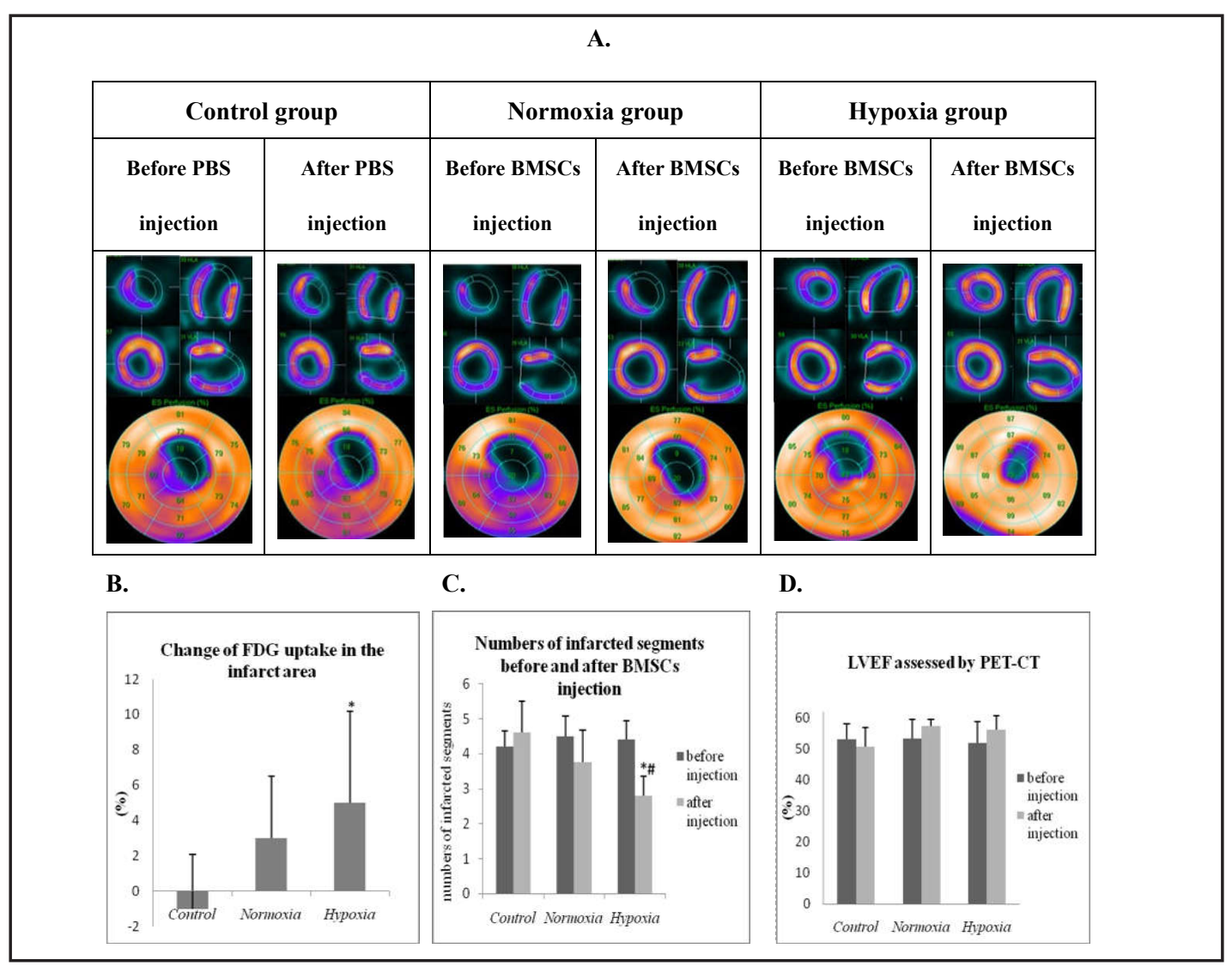

Fig. 3. (A) Images illustrating the different therapeutic effects (improvement of FDG uptake in the infarct zone and reduction of infarct area) following BMSC injection in all three groups. The improvement of FDG uptake in the infarct zone (B) and the reduction of infarct area (C) and was significantly enhanced in the hypoxia group, compared to the control group; while the differences between the normoxia group and the control group were not statistically significant. However, the left ventricular ejection fraction (D) was not significant different between groups, nor did it differ between pre- and post-BMSC administration. *, p $<0.05$ compared to the control group at the same time point; \#, $\mathrm{p}<0.05$ compared to before BMSC administration. 
Table 1. Hemodynamic parameters derived from pressure-volume relations. HR, heart rates; $\mathrm{CO}$, cardiac output; $\mathrm{EF}$, ejection fraction; $\mathrm{dP} / \mathrm{dT}_{\max }$, peak rate of pressure rise; - $\mathrm{dP} /$ $\mathrm{dT}_{\text {min' }}$, peak rate of pressure decline; SW, stroke work; $\mathrm{E}_{\text {max }}$, arterial elastance; PRSW, preload recruited stroke work. *, p <0.05 compared with control group; \#, p <0.05 compared with Normoxia group

\begin{tabular}{lccc} 
parameters & Control group & Normoxia group & Hypoxia group \\
\hline $\mathrm{HR}(/ \mathrm{bpm})$ & $265.8 \pm 22.1$ & $258.8 \pm 29.5$ & $262 \pm 11.3$ \\
$\mathrm{CO}(\mathrm{mL} / \mathrm{min})$ & $37.4 \pm 11.4$ & $48.5 \pm 17.6$ & $54.7 \pm 17.9$ \\
$\mathrm{EF}(\%)$ & $52.8 \pm 6.8$ & $56.8 \pm 3.9$ & $58.3 \pm 6.2$ \\
$\mathrm{dP} / \mathrm{dT}$ max $(\mathrm{mmHg} / \mathrm{s})$ & $4598.2 \pm 1784.4$ & $5474 \pm 864.3$ & $5709.6 \pm 1130.0$ \\
$-\mathrm{dP} / \mathrm{dT}_{\min }(\mathrm{mmHg} / \mathrm{s})$ & $-3590.6 \pm 1477.3$ & $-4341.5 \pm 112.1$ & $-4528.6 \pm 891.4$ \\
$\mathrm{SW}(\mathrm{mmHg} . \mathrm{mL})$ & $10341 \pm 4042.1$ & $12153 \pm 3776.3$ & $14787.8 \pm 3950$ \\
$\mathrm{Emax}$ & $2.6 \pm 0.8$ & $3.3 \pm 1.4$ & $3.6 \pm 1.1$ \\
$\mathrm{dP} / \mathrm{dT} \mathrm{T}_{\max . \mathrm{EDV}}(\mathrm{mmHg} / \mathrm{s} . \mu \mathrm{L})$ & $29.8 \pm 6.6$ & $37.3 \pm 9.5$ & $65.1 \pm 15.1 * \#$ \\
$\mathrm{PRSW}(\mathrm{mmHg})$ & $45.9 \pm 17.4$ & $66.4 \pm 26.6$ & $73.8 \pm 25.5 *$ \\
\hline
\end{tabular}

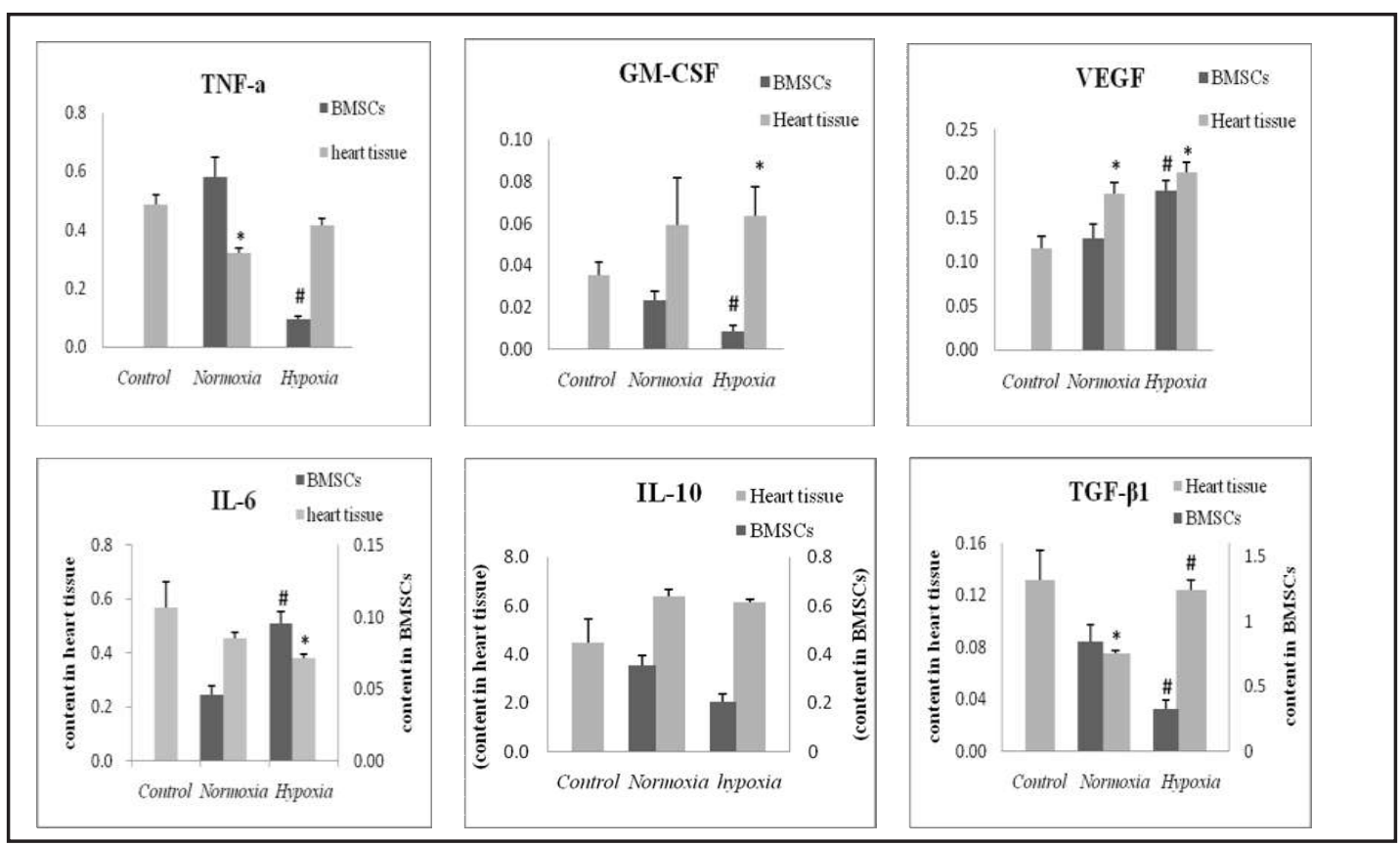

Fig. 4. Bio-Plex assay revealing the different cytokine expression spectrum in BMSCs cultured in normoxia and hypoxia, and in heart tissue. TNF, tumor necrosis factor; GM-CSF, granulocyte macrophage colony stimulating factor; TGF, transforming growth factor; IL, interleukine; VEGF, vascular endothelial growth factor. $*, p<0.05$, compared to the control group; \#, p <0.05, compared to the normoxia group. The unit for the Ordinates is $\mu \mathrm{g} / \mathrm{mg}$ of total protein.

culture significantly inhibited the expression of pro-inflammatory cytokines TNF- $\alpha$ and GM-CSF, and pro-fibrotic cytokine TGF- $\beta 1$, as well as promoting the production of antiinflammatory cytokine IL- 6 and angiogenic cytokine VEGF (all $p<0.05$ ). A trend was seen in the production of another anti-inflammatory cytokine IL-10, increasing in hypoxic culture, although the difference was not significant between two conditions. In in vivo set-up, similar changes in heart tissue cytokine expressions were documented in normoxia and hypoxia

\section{KARGER}


Fig. 5. (A) Histomorphometric exams with Hematoxylin-EosinSaffron (HES) staining, Picrosirius Red staining and CD31 antibody staining for the assessment of inflammatory infiltration as well as the extent of fibrosis, total collagen coontent, and neovessel formation, respectively. Magnification was $\times 100$. (B) Co m p a red with control group, normoxia and hypoxia groups showed a trend of decreased inflammation, but the differences between groups were not significant. However, the reduction of myocardial fibrosis (C) and neovascularization (D)

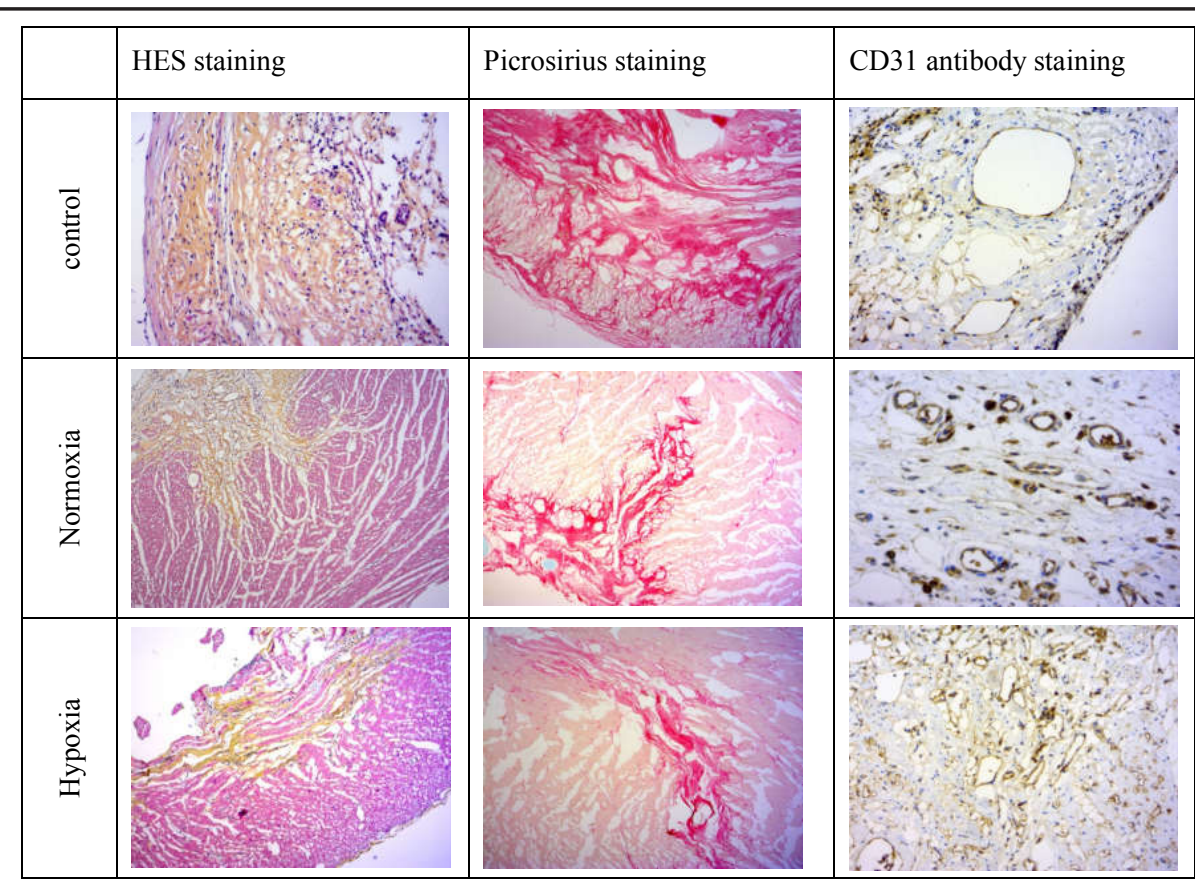

B.

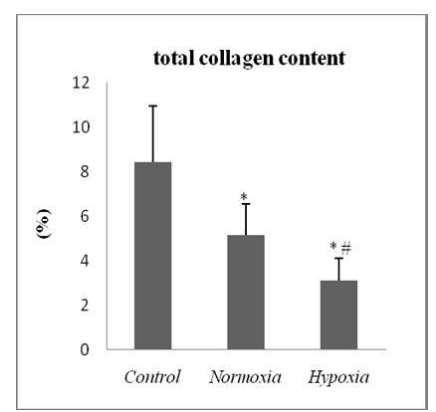

D.

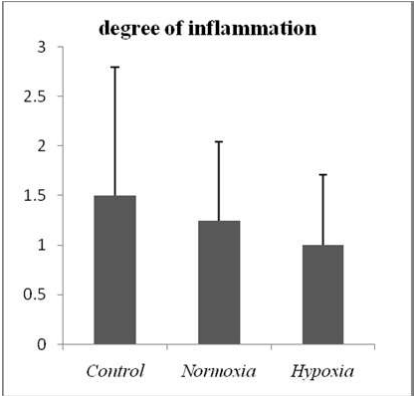

C.

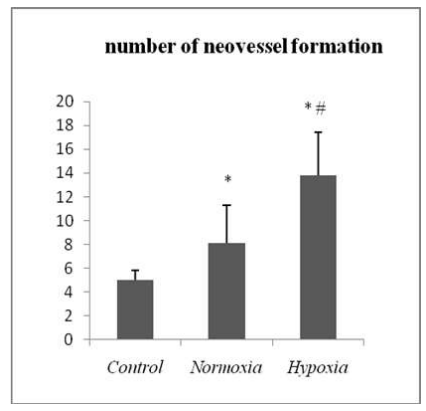

E.

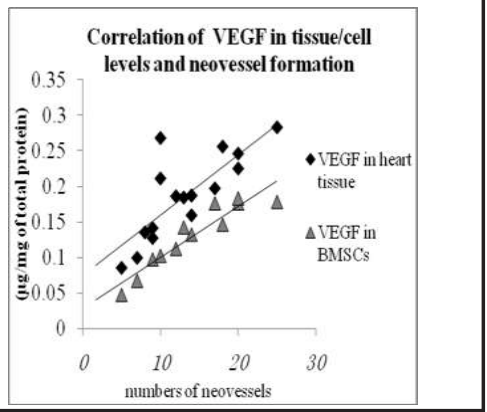

were significantly favored by intramyocardial injection of BMSCs, especially when cultured in hypoxia. (E) There was a linear correlation between VEGF concentrations and neovessel formation, in both tissue level ( $r=0.79)$ and cell level $(\mathrm{r}=0.84) .{ }^{*}, \mathrm{p}<0.05$ compared with the control group; \#, $\mathrm{p}<0.05$ compared with the normoxia group.

groups when compared to control group, with increased GM-CSF and VEGF expression, and decreased TNF- $\alpha$, TGF- $\beta 1$ and IL- 6 expression. The expression of TGF- $\beta 1$ was significantly higher in hypoxia group than normoxia group; however, no significant difference was evidenced in other cytokine expression between two groups.

\section{KARGER}


Hypoxia-pretreated BMSCs reduced myocardial inflammation and fibrosis, and promoted angiogenesis in the infarcted myocardium

As demonstrated by Fig. 5, HES staining revealed less interstitial inflammatory cell infiltration in the normoxia $(1.2 \pm 0.7,[0,2])$ and hypoxia $(1.0 \pm 0.7,[0,1])$ groups compared to the control group $(1.5 \pm 1.2,[0,3])$, yet the difference was not significant among them. Picrosirius Red staining demonstrated that intramyocardial injection of BMSCs was associated with a reduction in the total collagen content which was $8.4 \pm 4.5 \%, 5.1 \pm 3.1 \%$ and $3.1 \pm 1.6 \%$ in control, normoxia and hypoxia groups, respectively $(p<0.05)$. Neovessel numbers revealed by CD31 antibody staining were $5.0 \pm 0.8,8.1 \pm 3.1$ and $13.8 \pm 3.6$ in control, normoxia and hypoxia groups, respectively $(p<0.05)$. A linear correlation was observed between the neovessel formation and the VEGF levels in the BMSCs ( $\mathrm{r}=0.84, p<0.05)$, as well as those in heart tissues $(\mathrm{r}=0.79, p<0.05)$.

\section{Discussion}

In chronic MI, particularly when transmural necrosis is involved, focal inflammatory responses, massive fibrotic ongoing processes, an inexorable loss of contractile cells and angiogenesis occur, inducing profound secondary lesion enlargement, reducing contractility, and negatively impacting clinical outcomes [31,32]. By combining in vitro and in vivo multimodal evaluation, the principal findings of the current study were that one month hypoxia culture of rat BMSCs (i) significantly enhanced these cells' clonogenic potential and proliferation without inducing changes in their multipotency; and (ii) improved myocardial viability and contractility probably by modulating immune/inflammation status and promoting angiogenesis.

In line with previous publications $[9,17,33]$, our study demonstrated that hypoxic culture of BMSCs, compared to normoxic culture, vastly accelerated in vitro cell proliferation, with increased fibroblast colony-forming units and decreased population-doubling time. The cell phenotype and surface markers remained stable in each passage, and were comparable between the two culture conditions, signifying that cell stemness, namely the self-renewal and multipotency, was well preserved. We believe that conducting permanent hypoxic culture instead of transient hypoxic preconditioning represents a more favorable condition for BMSC in vitro expansion and selection. One of the rationales for using hypoxic culture is that the mesenchymal stem cell niche is low in 02 concentration, and in vitro expansion under "physiological" hypoxia helps reduce the oxidative stress and decrease the production of reactive oxygen species by suppressing mitochondrial respiration [34]. Moreover, the harsh microenvironment in the infarcted zone represents an obstacle for cell-based cardiac repair: the ongoing inflammation, deleterious oxidative stress, and lack of oxygen and nutrients impede the engraftment and survival of BMSCs. In vitro hypoxic culture could enhance their anaerobic metabolic activity and capacity to adapt to a hostile microenvironment, thus potentially improves the BMSC survival after implantation, and further enhances their therapeutic potentials $[9,13]$.

Our study further illustrated that hypoxic culture of BMSCs could modify the expression of cytokines in such a way as to promote modulation in inflammatory and in fibrosis process. As the Bio-Plex assay revealed, BMSCs cultured in hypoxia, compared to those cultured in normoxia, released significantly less pro-inflammatory cytokines (TNF- $\alpha$, GM-CSF), more anti-inflammatory cytokines (IL-6, IL-10), and less pro-fibrotic cytokine (TGF- $\beta 1$ ). Of particular interest, our data confirmed that hypoxic condition potentiated the production VEGF as it was also evidenced elsewhere $[35,36]$. However, the inflammation network and cytokine cascades in myocardial infarction might, in fact, elaborate temporally and spatially in a complicated and dynamic pattern [32]. This might render very challenging any illustration of the precise mechanisms underlying the beneficial changes of cytokine expression provided by in situ interaction between engrafted cells (cultured in hypoxia or not) and native hypoxic tissues. Indeed, in chronic MI, profound changes in ventricular architecture as well as dense collagen deposition might then represent a serious obstacle. 
The dynamic inflammatory response and cytokine elaboration following myocardial infarction are reported to be highly significant in the healing process as well as in the detrimental ventricular remodeling [32]. Previous studies have therefore demonstrated that mechanisms mediating cell-based cardiac repair might involve immunomodulation and antiinflammatory pathways [37]. In our experiment, our in vivo data provided more complex patterns for tissue's cytokine variations than that observed in in vitro set-up. In hypoxia group, the balance of expression of GM-CSF/IL- 6 seemed to indicate a modulation of inflammation (down regulation of tissue IL-6) in keeping with an upregulated immunomodulator cytokine GM-CSF. On the other hand, expression of VEGF and TGF- $\beta 1$ was also upregulated. Recently, there was a growing body of evidence involving VEGF and TGF- $\beta 1$ and their regulation though respectively PI3K/Akt and TGF- $\beta$ /SMAD2 pathways in the wound healing process i.e. modulation in collagen expression of cardiac fibroblasts [38].

Another main finding that emanated from our study was that long-term hypoxic preconditioned BMSCs produced marked increase in tissue viability and in cardiac function compared to their counterpart raised in conventional normoxic medium. The beneficial effect of BMSCs in chronic myocardial infarct areas was somewhat expected as we have already shown in our previous investigation. Indeed, outcomes from not only preclinical [39] but also in clinical setup [40] have demonstrated that (i) enhancing viability was one key role of cell therapy in chronic MI, and (ii) when associated with coronary artery bypass grafting procedures, cell therapy was able to boost the myocardial perfusion and viability in the MI areas, indicating the importance of angiogenic mechanism. In this study, regional viability was higher when MI segments were treated with hypoxic preconditioned BMSCs. In addition, we found that among the growth factors and cytokines produced by hypoxic BM-MSCs, VEGF was perhaps the most relevant to MI repair since a strong correlation could be found between the levels of in vitro/in vivo VEGF production and the quantity of neovessel formation in the infarcted zone as revealed by immunohistochemical findings. It has been demonstrated in a rat massive hepatectomy model that administration of hypoxic preconditioned BMSCs was associated with significant elevation of VEGF level in liver homogenate while this effect was blocked by perioperative injection of VEGF neutralized antibody [15]. It is noteworthy that other works have also documented that hypoxia promoted changes in glucose metabolism of MSCs and the latter observation could support the enhancement of FDG uptake evidenced here [41]. Taken together, since hypoxia is an important stimulus promoting the secretion of VEGF; our data here underlines that VEGF-mediated angiogenesis might be one of the underlying mechanisms of BMSC-based cardiac repair, through which BMSCs cultured in hypoxia, rather than normoxia, are associated with better therapeutic effects.

According to Millar cardiac catheter examination, although there was a trend toward an improvement in both normoxia- and hypoxia-BMSC treated animals, there were no significant differences in $\mathrm{EF}, \mathrm{dP} / \mathrm{dtmax}, \mathrm{dP} / \mathrm{dtmin}, \mathrm{SW}$, and Emax when compared to vehicle group. In contrast, a clear improvement in load-independent parameters of $\mathrm{LV}$ performance was documented with dP/dTmax.EDV and with PRSW in animals that experienced intramyocardial implantation of hypoxic preconditioned BMSCs. According to our experience with regard to the management of chronic MI, it is very difficult, albeit this is the ultimate goal, to significantly reverse myocardial functional impairments. As it was also demonstrated in our previous studies [42], except in case of intermediate infarction where a residual metabolism still persisted, marked myocardial perfusion and/or viability were accompanied by a modest improvement in global function.

However, the exact mechanism of improvement of tissue viability, perfusion and architecture induced by close interaction between hypoxic selected cells and hypoxic MI environment was not completely clear-cut herein. We hypothesize, in keeping with the positive outcomes from implanted cells on fibrosis, on infarct size and on neoangiogenesis, that such tissue characteristics in cytokines might (i) influence further wound healing process of MI tissue and (ii) improve the contractile components of MI hearts. However, our study holds several limitations. We did not perform cell tracking procedures so that survival rates and direct role of engrafted cells in repair process were not available. We have developed an 


\section{Cellular Physiology Cell Physiol Biochem 2017;44:1064-1077 \begin{tabular}{l|l|l} 
and Biochemistry Published online: November 24, 2017 & $\begin{array}{l}\text { (c) } 2017 \text { The Author(s). Published by S. Karger AG, Basel } \\
\text { www.karger.com/cpb }\end{array}$
\end{tabular}}

Liu et al.: Hypoxia and Cellular Therapy

isotopic labelling that allowed cell follow-up in vivo but it was efficient only for a very short period, i.e. 7 days [27]. Long-term cell tracking is still a challenging technique that requires both development and careful examination.

\section{Conclusion}

Our results were reported to be strongly supportive of the hypothesis that matching the environment in cell therapy, i.e. conducting long-term preconditioning or selection of BMSCs in low oxygen tension conditions resembling those observed in the chronic infarct area, might favor the cooperative activity of specific cytokines and may enhance the cardioprotective/ reparative role of these BMSCs when implanted in chronic MI. The observed beneficial impact on myocardial perfusion and contractility can potentially be ascribed to pathways involving the modulation of tissue inflammation and promotion of angiogenesis.

\section{Acknowledgements}

This work was funded by the National Natural Science Foundation of China (81600229) and Special Funds for Fundamental Scientific Research in Central Universities (011853031).

\section{Disclosure Statement}

The authors have no conflict of interest to declare.

\section{References}

1 Liu J, Wu P, Wang H, Wang Y, Du Y, Cheng W, Xu Z, Zhou N, Wang L: Necroptosis Induced by Ad-HGF Activates Endogenous C-Kit+ Cardiac Stem Cells and Promotes Cardiomyocyte Proliferation and Angiogenesis in the Infarcted Aged Heart. Cell Physiol Biochem 2016;40:847-860.

-2 Ludwig M, Tölk A, Skorska A, Maschmeier C, Gaebel R, Lux CA, Steinhoff G, David R: Exploiting AT2R to Improve CD117 Stem Cell Function In vitro and In vivo--Perspectives for Cardiac Stem Cell Therapy. Cell Physiol Biochem 2015;37:77-93.

3 Tongers J, Losordo DW, Landmesser U: Stem and progenitor cell-based therapy in ischaemic heart disease: promise, uncertainties, and challenges. Eur Heart J 2011;32:1197-1206.

4 Rosen MR, Myerburg RJ, Francis DP, Cole GD, Marbán E: Translating Stem Cell Research to Cardiac Disease Therapies. J Am Coll Cardiol 2014;64:922-937.

5 Williams AR, Hare JM: Mesenchymal Stem Cells: Biology, Pathophysiology, Translational Findings, and Therapeutic Implications for Cardiac Disease. Circ Res 2011;109:923-940.

-6 Haque N, Rahman MT, Abu Kasim NH, Alabsi AM: Hypoxic Culture Conditions as a Solution for Mesenchymal Stem Cell Based Regenerative Therapy. Sci World J 2013;2013:1-12.

7 Rosová I, Dao M, Capoccia B, Link D, Nolta JA: Hypoxic Preconditioning Results in Increased Motility and Improved Therapeutic Potential of Human Mesenchymal Stem Cells. Stem Cells 2008;26:2173-2182.

-8 Mohyeldin A, Garzón-Muvdi T, Quiñones-Hinojosa A: Oxygen in Stem Cell Biology: A Critical Component of the Stem Cell Niche. Cell Stem Cell 2010;7:150-161.

-9 Tsai C-C, Yew T-L, Yang D-C, Huang W-H, Hung S-C: Benefits of hypoxic culture on bone marrow multipotent stromal cells. Am J Blood Res 2012;2:148-159.

-10 Cicione C, Muiños-López E, Hermida-Gómez T, Fuentes-Boquete I, Díaz-Prado S, Blanco FJ: Effects of Severe Hypoxia on Bone Marrow Mesenchymal Stem Cells Differentiation Potential. Stem Cells Int 2013;2013:1-11. 


\section{Cellular Physiology Cell Physiol Biochem 2017;44:1064-1077 \begin{tabular}{l|l|l} 
and Biochemistry $10.1159 / 000485406$ & $\begin{array}{l}\text { D 2 } 2017 \text { The Author(s). Published by S. Karger AG, Basel } \\
\text { www.karger.com/cpb }\end{array}$ \\
\hline
\end{tabular}}

Liu et al.: Hypoxia and Cellular Therapy

11 Holzwarth C, Vaegler M, Gieseke F, Pfister SM, Handgretinger R, Kerst G, Muller L: Low physiologic oxygen tensions reduce proliferation and differentiation of human multipotent mesenchymal stromal cells. BMC Cell Biol 2010;11:11. DOI:10.1186/1471-2121-11-11.

12 Fehrer C, Brunauer R, Laschober G, Unterluggauer H, Reitinger S, Kloss F, Gully C, Gassner R, Lepperdinger G: Reduced oxygen tension attenuates differentiation capacity of human mesenchymal stem cells and prolongs their lifespan. Aging Cell 2007;6:745-757.

-13 Estrada JC, Albo C, Benguría A, Dopazo A, López-Romero P, Carrera-Quintanar L, Roche E, Clemente EP, Enriquez JA, Bernad A, Samper E: Culture of human mesenchymal stem cells at low oxygen tension improves growth and genetic stability by activating glycolysis. Cell Death Differ 2012;19:743-755.

14 Ohnishi S, Yasuda T, Kitamura S, Nagaya N: Effect of hypoxia on gene expression of bone marrow-derived mesenchymal stem cells and mononuclear cells. Stem Cells Dayt Ohio 2007;25:1166-1177.

-15 Yu J, Yin S, Zhang W, Gao F, Liu Y, Chen Z, Zhang M, He J, Zheng S: Hypoxia preconditioned bone marrow mesenchymal stem cells promote liver regeneration in a rat massive hepatectomy model. Stem Cell Res Ther 2013;4:83. DOI:10.1186/scrt234.

16 Leroux L, Descamps B, Tojais NF, Séguy B, Oses P, Moreau C, Daret D, Ivanovic Z, Boiron JM, Lamaziere JM, Dufourcq P, Couffinhal T, Duplaa C: Hypoxia Preconditioned Mesenchymal Stem Cells Improve Vascular and Skeletal Muscle Fiber Regeneration After Ischemia Through a Wnt4-dependent Pathway. Mol Ther 2010;18:1545-1552.

17 Basciano L, Nemos C, Foliguet B, de Isla N, de Carvalho M, Tran N, Dalloul A: Long term culture of mesenchymal stem cells in hypoxia promotes a genetic program maintaining their undifferentiated and multipotent status. BMC Cell Biol 2011;12:12, DOI:10.1186/1471-2121-12-12.

18 Song S-W, Kim K-E, Choi J-W, Lee CY, Lee J, Seo H-H, Lim KH, Lim S, Kim SW, Hwang KC: Proteomic Analysis and Identification of Paracrine Factors in Mesenchymal Stem Cell-Conditioned Media under Hypoxia. Cell Physiol Biochem 2016;40:400-410.

19 Buravkova LB, Andreeva ER, Gogvadze V, Zhivotovsky B: Mesenchymal stem cells and hypoxia: Where are we? Mitochondrion 2014;19:105-112.

20 Uemura R: Bone Marrow Stem Cells Prevent Left Ventricular Remodeling of Ischemic Heart Through Paracrine Signaling. Circ Res 2006;98:1414-1421.

-21 Hu X, Yu SP, Fraser JL, Lu Z, Ogle ME, Wang J-A, Wei L: Transplantation of hypoxia-preconditioned mesenchymal stem cells improves infarcted heart function via enhanced survival of implanted cells and angiogenesis. J Thorac Cardiovasc Surg 2008;135:799-808.

-22 Hu X, Xu Y, Zhong Z, Wu Y, Zhao J, Wang Y, Cheng H, Kong M, Zhang F, Chen Q, Sun J, Li Q, Jin J, Li Q, Chen L, Wang C, Zhan H, Fan Y, Yang Q, Yu L, Wu R, Liang J, Zhu J, Wang Y, Jin Y, Lin Y, Yang F, Jia L, Zhu W, Chen J, Yu H, Zhang J, Wang J: A Large-Scale Investigation of Hypoxia-Preconditioned Allogeneic Mesenchymal Stem Cells for Myocardial Repair in Nonhuman Primates. Circ Res 2016;118:970-983.

23 Wang J, He A, Hu X, Jiang Y, Sun Y, Jiang J, Gui C, Wang Y, Chen H: Anoxic preconditioning: a way to enhance the cardioprotection of mesenchymal stem cells. Int J Cardiol 2009;133:410-412.

-24 Teng X, Chen L, Chen W, Yang J, Yang Z, Shen Z: Mesenchymal Stem Cell-Derived Exosomes Improve the Microenvironment of Infarcted Myocardium Contributing to Angiogenesis and Anti-Inflammation. Cell Physiol Biochem 2015;37:2415-2424.

25 Zhang J, Chen A, Wu Y, Zhao Q: Placental Growth Factor Promotes Cardiac Muscle Repair via Enhanced Neovascularization. Cell Physiol Biochem 2015;36:947-955.

-26 Li C, Wu X, Tong J, Yang X, Zhao J, Zheng Q Zhao GB, Ma ZJ: Comparative analysis of human mesenchymal stem cells from bone marrow and adipose tissue under xeno-free conditions for cell therapy. Stem Cell Res Ther 2015;6:55. DOI: 10.1186/s13287-015-0066-5.

27 Tran N, Li Y, Maskali F, Antunes L, Maureira P, Laurens M-H, Marie PY, Karcher G, Groubatch F, Stoltz JF, Villemot JP: Short-term heart retention and distribution of intramyocardial delivered mesenchymal cells within necrotic or intact myocardium. Cell Transplant 2006;15:351-358.

-28 Zhang Z, Yang C, Shen M, Yang M, Jin Z, Ding L, Jiang W, Yang J, Chen H, Cao F, Hu T: Autophagy mediates the beneficial effect of hypoxic preconditioning on bone marrow mesenchymal stem cells for the therapy of myocardial infarction. Stem Cell Res Ther 2017;8:89. DOI:10.1186/s13287-017-0543-0. 


\section{Cellular Physiology Cell Physiol Biochem 2017;44:1064-1077 \begin{tabular}{ll|l} 
and Biochemistry Published online: November 24, 2017 & $\begin{array}{l}\text { @ } 2017 \text { The Author(s). Published by S. Karger AG, Basel } \\
\text { www.karger.com/cpb }\end{array}$ \\
\hline
\end{tabular}}

Liu et al.: Hypoxia and Cellular Therapy

29 Dominici M, Le Blanc K, Mueller I, Slaper-Cortenbach I, Marini F, Krause D, Deans R, Keating A, prockop Dj, Horwitz E: Minimal criteria for defining multipotent mesenchymal stromal cells. The International Society for Cellular Therapy position statement. Cytotherapy 2006;8:315-317.

-30 Hadi AM, Mouchaers KTB, Schalij I, Grunberg K, Meijer GA, Vonk-Noordegraaf A, Van der Laarse WJ, Belien JA: Rapid quantification of myocardial fibrosis: a new macro-based automated analysis. Cell Oncol Dordr 2011;34:343-354.

-31 Zhang J, He Z, Xiao W, Na Q, Wu T, Su K, Cui X: Overexpression of BAG3 Attenuates Hypoxia-Induced Cardiomyocyte Apoptosis by Inducing Autophagy. Cell Physiol Biochem 2016;39:491-500.

-32 Nian M: Inflammatory Cytokines and Postmyocardial Infarction Remodeling. Circ Res 2004;94:1543-1553.

-33 Raheja LF, Genetos DC, Yellowley CE: The effect of oxygen tension on the long-term osteogenic differentiation and MMP/TIMP expression of human mesenchymal stem cells. Cells Tissues Organs 2010;191:175-184.

-34 Zhang Y, Marsboom G, Toth PT, Rehman J. Mitochondrial Respiration Regulates Adipogenic Differentiation of Human Mesenchymal Stem Cells. PLoS ONE 2013;8:e77077. Doi: 10.1371/journal.pone.0077077.

35 Saparov A, Chen C-W, Beckman S, Wang Y, Huard J: The Role of Antioxidation and Immunomodulation in Postnatal Multipotent Stem Cell-Mediated Cardiac Repair. Int J Mol Sci 2013;14:16258-16279.

-36 Zisa D, Shabbir A, Suzuki G, Lee T: Vascular endothelial growth factor (VEGF) as a key therapeutic trophic factor in bone marrow mesenchymal stem cell-mediated cardiac repair. Biochem Biophys Res Commun 2009;390:834-838.

-37 Xu M, Uemura R, Dai Y, Wang Y, Ashraf M: In vitro and in vivo effects of bone marrow stem cells on cardiac structure and function. J Mol Cell Cardiol 2007;42:441-448.

-38 Jun EK, Zhang Q Yoon BS, Moon J-H, Lee G, Park G, Kang PJ, Lee JH, Kim A, You S: Hypoxic conditioned medium from human amniotic fluid-derived mesenchymal stem cells accelerates skin wound healing through TGF- $\beta$ /SMAD2 and PI3K/Akt pathways. Int J Mol Sci 2014;15:605-628.

-39 Tran N, Franken PR, Maskali F, Nloga J, Maureira P, Poussier S, Groubatch F, Vanhove C, Villemot JP, Marie PY: Intramyocardial Implantation of bone marrow-derived stem cells enhances perfusion in chronic myocardial infarction: dependency on initial perfusion depth and follow-up assessed by gated pinhole SPECT. J Nucl Med 2007;48:405-412.

40 Hu X, Huang X, Yang Q, Wang L, Sun J, Zhan H, Lin J, Pu Z, Jiang J, Sun Y, Xiang M, Liu X, Xie X, Yu X, Chen Z, Tse HF, Zhang J, Wang J: Safety and efficacy of intracoronary hypoxia-preconditioned bone marrow mononuclear cell administration for acute myocardial infarction patients: The CHINA-AMI randomized controlled trial. Int J Cardiol 2015;184:446-451.

41 Miyamoto M, Kuroda S, Zhao S, Magota K, Shichinohe H, Houkin K, Kuge Y, Tamaki N: Bone marrow stromal cell transplantation enhances recovery of local glucose metabolism after cerebral infarction in rats: a serial 18F-FDG PET study. J Nucl Med 2013;54:145-150.

42 Maureira P, Marie P-Y, Liu Y, Yu F, Poussier S, Maskali F, Groubatch F, Karcher G, Tran N: Sustained therapeutic perfusion outside transplanted sites in chronic myocardial infarction after stem cell transplantation. Int J Cardiovasc Imaging 2013;29:809-817. 